\title{
Clinical and Radiological Comparison between Ipsilateral and Contralateral Side Canal Decompression Using an Unilateral Laminotomy Approach
}

\author{
Woong Bae Park, Jae Taek Hong, Sang Won Lee, Jae Hoon Sung, \\ Seung Ho Yang, IL Sub Kim \\ Department of Neurosurgery, St. Vincent Hospital, The Catholic University of Korea College of Medicine, Suwon, Korea
}

Objective: To compare the clinical and radiological outcome of both sides using the unilateral approach.

Methods: Unilateral laminotomy was performed to achieve bilateral decompression. Thirty-nine patients who underwent this procedure were analyzed prospectively using the Oswestry Disability Index (ODI), the visual analog scale (VAS) pain score to evaluate symptoms in both legs, and the radiological morphometric index to calculate the anteriorposterior diameter and midcanal width. The incidence of complications from this approach was then evaluated.

Results: The mean follow-up time was 12.2 months. The mean ODI was 48.4 preoperatively and 14.2 postoperatively. The mean dural sac widening of the ipsilateral side $(187.0 \%)$ was significantly larger $(p<0.01)$ than that of the contralateral side $(145.6 \%)$. The VAS improvement ratio ([preoperative VAS score-postoperative VAS score]/[preoperative VAS score] $\times 100)$ for the pain in each leg was $75.4 \%$ (ipsilateral side) and $73.7 \%$ (contralateral side). While the VAS improvement ratio for pain in each side was significantly reduced, the difference in the VAS ratio between sides was statistically insignificant $(p=0.64)$. There were 2 cases $(5.1 \%)$ of dural tearing during the procedure, 1 case $(2.6 \%)$ of transient paresthesia of nerve roots, and 2 cases $(5.1 \%)$ of transient paresthesia of the contralateral nerve root. The transient paresthesias of nerve roots never lasted more than 2 weeks.

Conclusion: This technique allows for significant decompression of the contralateral canal and excellent clinical outcomes without troublesome complications. Although ipsilateral the dural sac widening was significantly larger than contralateral side, the difference in the clinical outcome between sides was statistically insignificant.

Key Words: Spinal stenosis $\cdot$ Decompression $\cdot$ Outcome $\cdot$ Complication

\section{INTRODUCTION}

Laminectomy has been widely used in the treatment of lumbar spinal stenosis ${ }^{15,18)}$. Destruction of the spinal bony and ligamentous structures with concomitant instability and peridural scar formation are the main problems with this procedure $^{5,19,20)}$. To overcome these significant disadvantages, many procedures have been proposed. Technical variations of lumbar laminectomy include unilateral laminotomy, bilateral laminotomies, and open-door type laminoplasty ${ }^{2}$. However, they have never become widespread due to the complicated nature of these procedures and also a lack of follow-up data related to postoperative stability. Without clinical and radiological

- Received: January 27, 2016 • Revised: March 21, 2016

- Accepted: April 18, 2016

Corresponding Author: Jae Taek Hong

Department of Neurosurgery, St. Vincent Hospital, The Catholic

University of Korea College of Medicine, 93 Jungbu-daero, Paldal-gu, Suwon 16247, Korea

Tel: +82-31-249-8171, Fax: +82-31-249-5208

E-mail: jatagi15@gmail.com

$\otimes$ This is an open access article distributed under the terms of the Creative Commons Attribution Non-Commercial License (http://creativecommons.org/licenses/by-nc/4.0/) which permits unrestricted non-commercial use, distribution, and reproduction in any medium, provided the original work is properly cited. data demonstrating adequate decompression of the spinal canal and adequate postoperative follow-up to ensure an acceptable clinical outcome, widespread use of any new technique for lumbar decompression will not occur. Also, although ipsilateral canal decompression by laminotomy has been reported to be effective in the literature $1,3,9,11)$, no reports have analyzed the clinical and radiological results of the contralateral side following this procedure. So, the purpose of this study was to investigate the efficacy of unilateral laminotomy on both ipsilateral and contralateral decompression and compares the clinical and radiological outcome of both sides using this unilateral approach. The incidence of complications from this approach was then evaluated.

\section{MATERIALS AND METHODS}

\section{Patient Population}

We retrospectively reviewed the chart and radiological study and included the patient who underwent ipsilateral and bilateral decompression for lumbar stenosis and also performed postoperative resonance imaging (MRI) or myelography computed tomography (CT). All patients who had all of the following criteria were included in this study: (1) symptoms 
of bilateral neurogenic claudication referable to the lumbar spine (claudication or radicular symptoms brought on either by walking or by prolonged standing, relieved by sitting or a flexed position, and occurring in the absence of vascular or neuropathic pathology), (2) radiological/neuroimaging evidence of degenerative lumbar stenosis (i.e., neurologic compression by hypertrophied [infolded] ligamentum flavum, osteophytic facet joints, and annular bulging), (3) failure of conservative measures for at least 3 months, (4) absence of associated pathology such as instability, inflammation or malignancy, and (5) no history of surgery for lumbar stenosis or lumbar fusion. Patients presenting with mild degenerative spondylolisthesis (i.e., grade I spondylolisthesis without dynamic instability) were not excluded. Thirty-nine patients with degenerative lumbar spinal stenosis was enrolled in this study. All 39 subjects exhibited either mono- or multisegmental lumbar stenosis and had been suffering from bilateral leg claudication. They were treated for bilateral canal decompression by unilateral laminotomy, and they were followed for about 12 months. Twenty-eight patients demonstrated monosegmental stenosis while eleven patients had multilevel stenosis. Therefore, there were a total of 50 individual stenotic regions: L5-S1 stenosis in 3 patients, $\mathrm{L} 4-5$ stenosis in 30 patients, $\mathrm{L}^{-}-4$ in 12 patients, L2 -3 in 4 patients, stenosis one case of L1-2 stenosis.

\section{Surgical Procedure}

General endotracheal anesthesia was used in all cases and patients were placed in gentle spinal flexion to provide widening of the interlaminar spaces. This position is identical to the one used for standard laminectomy. Under fluoroscopic guidance, the appropriate level for incision was determined. The initial incision was midline and a fascial incision was performed on the patient's most symptomatic side. Using a Cobb elevator, multifidus was elevated gently off the laminae at the involved segments, and then retracted to the medial border of the facet joint. A Taylor retractor then was utilized. The operating microscope was moved into the field, and the laminar edge was identified.

Using Kerrison rongeurs or a high speed burr, an ipsilateral laminotomy of the cephalad hemilamina was performed. It was extended superiorly until the insertion of the ligamentum flavum was reached. The microscope was then angulated into the ipsilateral subarticular zone and soft tissue and any bony stenosing pathology was excised using a high-speed drill and Kerrison rongeurs. Following ipsilateral complete decompression, the base of the spinous process and inferior surface of the contralateral lamina were undercut with a high-speed burr. Thinning of the contralateral laminae was performed prior to removal of the ligamentum flavum as this allows protection of the dura during bone work. By angling the microscopic view and tilting the surgical table towards the contralateral side after ipsilateral decompression, the contralateral ligamentum flavum was able to be resected for contralateral decompression. Contralateral ligamentum flavectomy and inner surface thinning of the contralateral lamina produced significant decompression of the dural sac that allowed for direct viewing of the contralateral facet. Finally, decompression of the contralateral subarticular recess was achieved by undercutting the ventromedial part of the facet with curved Kerrison rongeurs and a high-speed burr. Decompression was continued to the contralateral margin of the thecal sac, pedicle and contralateral nerve root. Exposure of the contralateral pedicle is critical to ensuring adequate decompression of the contralateral traversing nerve root.

After decompression of each stenotic area, the patient's position was changed into an extended position to confirm the adequacy of continued decompression in a functional position that would be most likely to cause foraminal stenosis. If there was any remaining pathology encroaching a nerve root, additional decompression procedure was performed with the patient's spine in in extended position.

\section{Outcome Measures}

Using the Oswestry Disability Index (ODI) score and visual analogue scale (VAS) pain score, clinical symptoms including the degree of pain in both legs and lower back pain were quantified before surgery, as well as at 1 month and 12 months after surgery to calculate improvement rates. Dural sac widening was evaluated using radiological morphometric indices (anteriorposterior diameter, width of ipsilateral and contralateral dural sac when divided in the midsagittal plane along the long axis of the spinous process and the center of the body) and then statistical analysis was performed on the data for each side and also to compare the 2 sides. Dural sac diameter was calculated by pre- and postoperative MRI or CT myelography (Figs. 1, 2). Postoperative MRI was checked 1 month after the surgery. CT myelography was checked instead of MRI in the cases with cardiac pacemaker. Anteriorposterior diameter was evaluated at the midsagittal area. Widths of ipsilateral side and contralateral side were checked at the most stenotic area by the ligamentum flavum and facet hypertrophy (usually just above the pedicle). Measurement of width was performed at the level of facet joint. Results were analyzed using the Wilcoxon signed-rank test.

To evaluate the presence of any correlations between postoperative radiological measurements and clinical outcomes, we compared the VAS improvement ratio ([preoperative VAS score-postoperative VAS score]/[preoperative VAS score $\times 100]$ ) of the pain in each leg and the dural sac widening ratio ([postoperative dural sac width-preoperative dural sac width]/[preoperative dural sac width $\times 100]$ ) on each side (ipsilateral side and contralateral side) of monosegmental spinal stenosis patients $(n=28)$ who had bilateral leg symptoms preoperatively. We followed up postoperative dynamic X-ray for all patients in every 3 months to check the postoperative instability. 


\section{RESULTS}

\section{Demographic Data}

The mean age of 39 patients was 65.3 years (range, 41-87 years). There were 19 men and 20 women. A total of 50 spinal segments were microscopically decompressed in the 39 patients. Twenty-eight patients had one-level decompression,
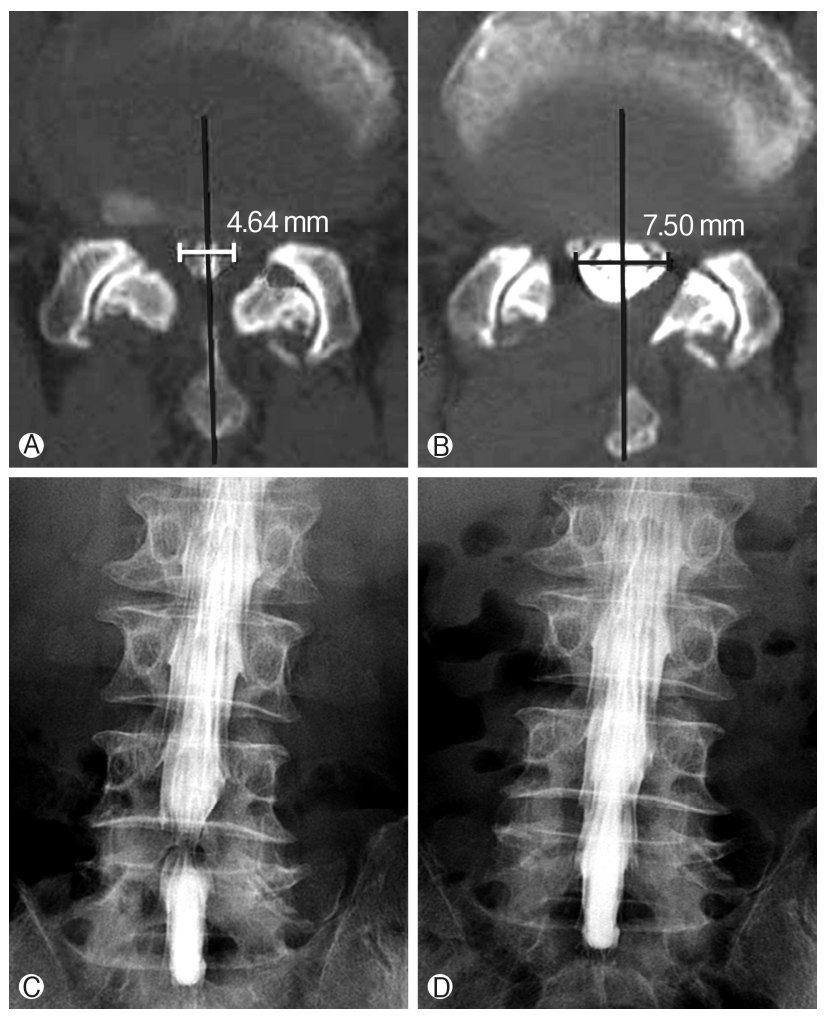

Fig. 1. Imaging studies (myelography and postmyelogram computed tomography) before (A, C) and after (B, D) surgery show marked enlargement of dural sac postoperatively. (A, B) The ipsilateral and contralateral dural sac boundaries are defined by the midsagittal plane along the spinous processes and centers of the body. Anteriorposterior diameter was evaluated at the midsagittal area. Widths of ipsilateral side and contralateral side were checked at the most stenotic area by the ligamentum flavum and facet hypertrophy (usually just above the pedicle). and 11 had multilevel decompressions. L4-5 and L3-4 levels were most commonly involved (Table 1). The mean follow-up time was about 12.2 months.

\section{Outcome of Surgery}

The anteriorposterior diameter of the dural sac was significantly increased bilaterally after surgery. The mean dural sac widening ipsilaterally was $187 \%$ and contralaterally $145.6 \%$ compared to the preoperative value resulting in a statistically significant p-value of less than 0.01 (Table 2).

Also, ipsilateral decompression required a substantially longer operating time than the contralateral side (38.9 minutes vs. 24.6 minutes). The contralateral side required less operating time because no paraspinal muscle dissection or laminotomy was performed on it.

There were 2 cases (5.1\%) of dural tearing during the proce-
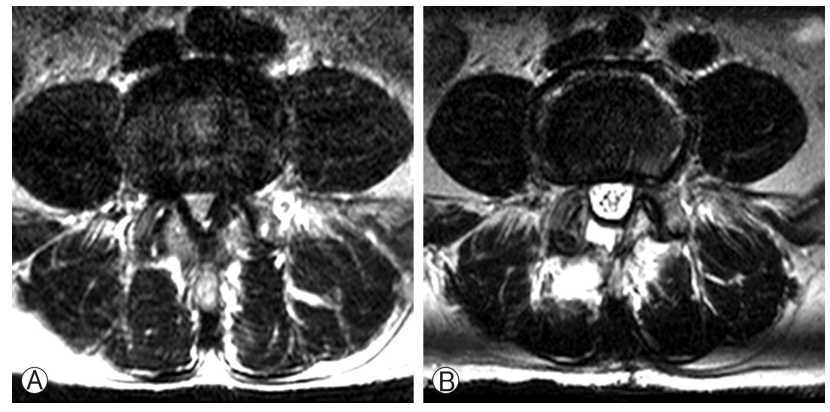

Fig. 2. (A) Preoperative axial magnetic resonance (MR) image shows significant narrowing of spinal canal by thickened ligamentum flavum and facet hypertrophy. (B) Postoperative MR image shows marked widening of the dural sac by the unilateral laminotomy and bilateral decompression technique.

Table 1. Distribution of spinal levels of lumbar spinal stenosis and surgical treatment

\begin{tabular}{cc}
\hline \hline Level & Laminotomy $(\mathrm{n}=50), \mathrm{n}(\%)$ \\
\hline L1-2 & $1(2)$ \\
L2-3 & $4(8)$ \\
L3-4 & $12(24)$ \\
L4-5 & $30(60)$ \\
L5-S1 & $3(6)$ \\
\hline
\end{tabular}

Table 2. Comparison of radiological parameters

\begin{tabular}{lccc}
\hline \multicolumn{1}{c}{ Parameter } & Preoperative & Postoperative & p-value \\
\hline Anteriorposterior diameter (mm) & 7.0 & 11.0 & $<0.01$ \\
Ipsilateral canal width (mm) & 4.1 & 7.1 & $<0.01$ \\
Contralateral canal width (mm) & 4.7 & 6.7 & $<0.01$ \\
Canal widening ratio (ipsilateral, \%) & 100 & 177.1 & $<0.01$ \\
Canal widening ratio (contralateral, \%) & 100 & 145.7 & $<0.01$ \\
\hline
\end{tabular}


dure without negative consequences. The dural tears occurred in 2 separate patients. One dural tear occurred ipsilaterally and the other contralaterally during the decompression procedure. The ipsilateral durotomy was primarily repaired with direct suture while the contralateral one was treated by gelfoam, fibrin glue and postoperative lumbar drainage because the size of the durotomy was small and the working space was not large enough to allow for primary repair.

Table 3. Surgical morbidities of bilateral canal decompression with using a unilateral approach

\begin{tabular}{lc}
\hline \hline Surgical morbidity & No. $(\%)$ \\
\hline Delayed instability & $1 / 39(2.6)$ \\
Dural tear & $2 / 39(5.1)$ \\
Transient paresthesia & $3 / 39(7.1)$ \\
Ipsilateral nerve root & $1 / 39(2.6)^{*}$ \\
Contralateral nerve root & $3 / 39(7.1)^{*}$ \\
\hline
\end{tabular}

"One patient experienced bilateral nerve root transient parasthesia.

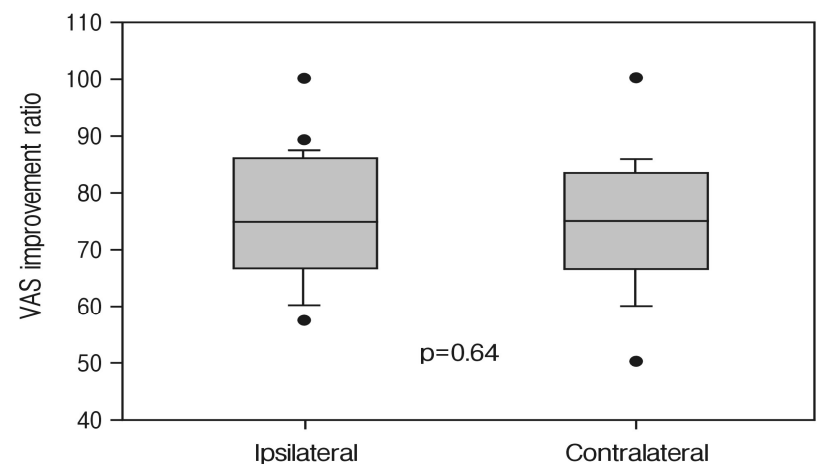

Fig. 3. Visual analog scale (VAS) improvement ratio for the pain in each leg was $75.4 \%$ (ipsilateral side) and $73.7 \%$ (contralateral side), which are both statistically insignificant $(p=0.64)$.

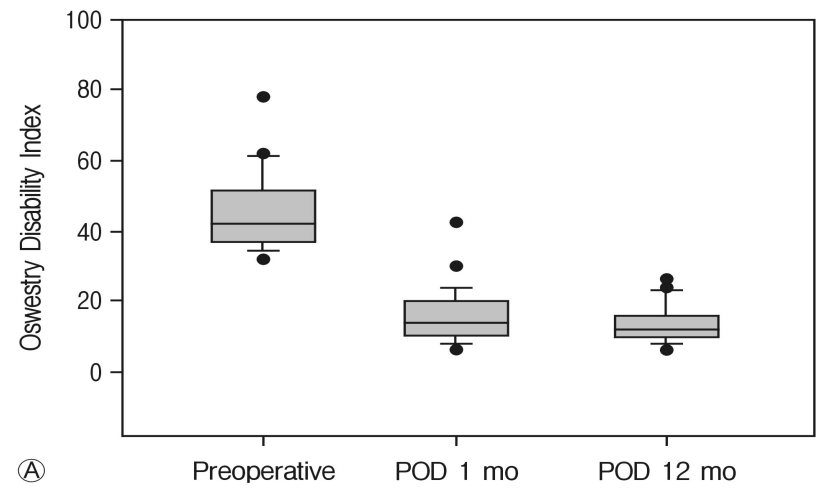

In this series, postoperative transient paresthesia was observed in 3 patients (Table 3): 1 case (2.6\%) of transient paresthesia of the nerve roots bilaterally and 2 cases (5.1\%) of transient paresthesia of the contralateral nerve root. Transient paresthesia of the nerve roots never lasted more than 2 weeks. Another patient developed mild instability during the follow-up period but no surgical intervention was needed because the patient's mild back pain was resolved with physiotherapy.

The mean follow-up time was 12.2 months. All patients improved clinically and radiologically after this procedure. The mean dural sac widening ratio of the ipsilateral side was $187.0 \%$ and of the contralateral side was $145.6 \%$, which was statistically significant $(p<0.01)$. However, the VAS improvement ratio ([preoperative VAS score-postoperative VAS score]/[preoperative VAS score] $\times 100$ ) for the pain in each leg was $75.4 \%$ for the ipsilateral side and $73.7 \%$ for the contralateral side. A significant decrease in the VAS improvement ratio for each side occurred. Importantly, the difference in postsurgical improvement between the 2 sides was statistically insignificant $(p=0.64)$ (Fig. 3). Therefore, the level of clinical improvement in both sides was statistically similar. Patients were also found to have signficant improvement in their postoperative ODI scores. There was no difference in the ODI improvement ratio between monosegmental and multisegmental spinal stenoses (Fig. 4). It was found that patients' postoperative ODI scores also correlated with postoperative improvement of their ipsilateral VAS scores $(\mathrm{r}=0.42, \mathrm{p}<0.05)$.

\section{DISCUSSION}

Bilateral spinal canal decompression with unilateral laminotomy has been proven to be effective in the literature and has become one of the primary treatment methods for lumbar stenosis $^{3,6,8,9,12)}$. However, no studies have compared the clinical and radiological efficacy between ipsilateral side and contralateral side after bilateral decompression using unilateral laminotomy.

One of the reasons for this gap in the literature is in the

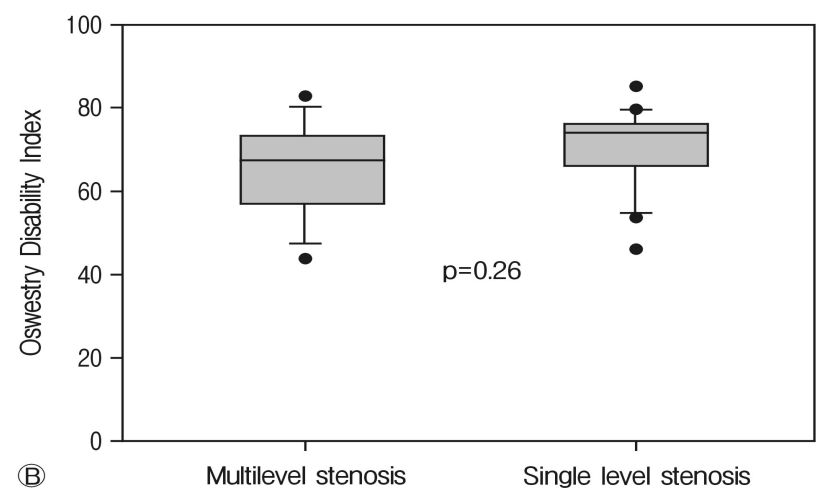

Fig. 4. (A) Time course of the overall Oswestry Disability Index-assessed functional score before surgery, at 1 month and 12 months postoperatively. (B) Measurement of the functional outcomes of mono-segmental and multisegmental stenoses demonstrated no significant differences. POD, postoperative. 
way that 2 commonly used pain assessment tools are utilized. The ODI and VAS scores are widely used to measure the overall level of patient discomfort. Usually, these evaluation tools are not used to distinguish between pain and disability on one side of the body. However, restricting the questions to each leg allows for a more accurate comparison of improvement pre- and postoperatively. This also can give a more detailed assessment of the efficacy of different procedures.

Therefore, there were several goals of this study that utilized patients who exhibited both lumbar stenosis and bilateral leg symptoms. First, we sought to compare the radiological and clinical outcomes of ipsilateral and contralateral decompression using unilateral laminotomy. Second, we wanted to evaluate potential differences in surgical time between ipsilateral and contralateral decompression using this unilateral technique. Third, to test the efficacy of this technique, we compared the morbidity of our data to previous reports using conventional decompression surgery.

There was a significant difference in dural sac widening of both the ipsilateral and contralateral sides, as well as a statistically significant difference in the degree of widening between the 2 sides $(\mathrm{p}<0.01)$ (Table 2). However, there was no significant statistical difference of the VAS score improvement ratio between the 2 sides (Fig. 3). This is important because it represents an important paradigm shift in the decision-making process for bilateral decompression.

Advances in radiological technology allows for better resolution and increasingly finer measurements ${ }^{4,7}$. For this reason, most spinal surgeons will use the radiological findings to choose which side to perform the unilateral laminotomy. However, we have found that in many patients with lumbar stenosis and bilateral leg claudication the severity of stenosis as detected radiologically does not always correlate with greater symptoms. In these patients, superior surgical resolution of symptoms is achieved by performing the laminotomy on the more symptomatic side regardless of the radiologic findings. If neither side is more symptomatic than the other, we have right-handed surgeons select the patient's left side for the incision since this allows for easier manipulation of surgical instruments.

Anatomically, the ipsilateral side lamina, ligamentum flavum, and medial side of the facet joint are removed while only the ligamentum flavum and ventromedial part of facet joint are removed on the contralateral side. Based on this study results, undercutting of a hypertrophied facet joint and removal of thickened ligamentum flavum on the contralateral side may be sufficient enough to decompress the neural structures and relieve symptoms while reducing postoperative inflammation, even in the presence of residual facet hypertrophy.

Thomé et al. ${ }^{17)}$ also reported a statistically significant increase in dural sac size after laminotomy or laminectomy but found no statistical relationship between the extent of decompression and clinical outcome.

Unintended durotomy is a common concern during spinal decompressive procedures. Overall, durotomy rates for laminectomy have been shown to range from $5 \%$ to $15 \%{ }^{11,13,14}$.
Bilateral laminotomy is complicated by dural tears less than $6 \%$ and unilateral laminotomy with contralateral decompression in $3.5 \%$ to $12 \%{ }^{10,16}$. In our series, the incidence of durotomy was $5.1 \%$, which is in accordance with the literature. Although there are perioperative concerns about dural tears, there is no association between dural tears and long-term sequelae.

Another concern for spine surgeons is that less invasive decompression techniques have been associated with an increased rate of neural injury. One potential explanation for this association is that less invasive surgeries usually involve a much more limited operating window and for less experienc ed surgeons this can be very challenging. According to our data, there was no permanent injury to any nerve roots. However, intraoperative manipulation and/or compression of nerve roots at that time may provoke transient radicular deficits, especially in the contralateral side (7.7\%).

Overall, our intra- and postoperative rates of complications were comparable to other surgical spinal decompression procedures such as laminectomy and bilateral laminotomy ${ }^{1,3,7,9,10,16}$. Even amongst symptomatic patients, no patient had symptoms lasting longer than 2 weeks. This refutes fears that the dural sac and nerve roots would be injured by using this unilateral approach.

There are some limitations to this study. The present study only analyzed patients who performed both preoperative and postoperative radiological study. So, there could be a selection bias. The limited number of study remains methodological concerns. Moreover, longer term follow-up study might be mandatory to conclude the effectiveness of this surgical technique. More research and larger volume of cases are needed to analyze the difference between ipsilateral and contralateral parameters.

\section{CONCLUSION}

Microscope-guided unilateral laminotomy is beneficial for bilateral decompression. Although ipsilateral the dural sac widening was significantly larger than contralateral side, this procedure adequately decompresses both the ipsilateral and contralateral sides, as demonstrated both radiologically and according to improvement of symptoms. Using this method, the integrity of the spinal bony and ligamentous structures are preserved, and a significant enlargement of the spinal canal can be achieved. Finally, because unilateral laminotomy is a minimally invasive procedure, it may be especially appropriate for weaker patients who are older or medically frail. Further long-term follow-up is needed to evaluate the continued spinal stability in these patients.

\section{CONFUCT OF INTEREST}

No potential conflict of interest relevant to this article was reported. 


\section{REFERENCES}

1. Arai Y, Hirai T, Yoshii T, Sakai K, Kato T, Enomoto M, et al: A prospective comparative study of 2 minimally invasive decompression procedures for lumbar spinal canal stenosis: unilateral laminotomy for bilateral decompression (ULBD) versus musclepreserving interlaminar decompression (MILD). Spine (Phila Pa 1976) 39:332-340, 2014

2. Chatani K: A novel surgical approach to the lumbar spine involving hemilateral split-off of the spinous process to preserve the multifidus muscle: technical note. J Neurosurg Spine 24:694699, 2016

3. Choi WS, Oh CH, Ji GY, Shin SC, Lee JB, Park DH, et al: Spinal canal morphology and clinical outcomes of microsurgical bilateral decompression via a unilateral approach for lumbar spinal canal stenosis. Eur Spine J 23:991-998, 2014

4. Dalgic A, Uckun O, Ergungor MF, Okay O, Daglioglu E, Hatipoglu G, et al: Comparison of unilateral hemilaminotomy and bilateral hemilaminotomy according to dural sac area in lumbar spinal stenosis. Minim Invasive Neurosurg 53:60-64, 2010

5. den Boogert HF, Keers JC, Marinus Oterdoom DL, Kuijlen JM: Bilateral versus unilateral interlaminar approach for bilateral decompression in patients with single-level degenerative lumbar spinal stenosis: a multicenter retrospective study of 175 patients on postoperative pain, functional disability, and patient satisfaction. J Neurosurg Spine 23:326-335, 2015

6. Deschuyffeleer S, Leijssen P, Bellemans J: Unilateral laminotomy with bilateral decompression for lumbar spinal stenosis: shortterm risks in elderly individuals. Acta Orthop Belg 78:672-677, 2012

7. Hong SW Choi KY, Ahn Y, Baek OK, Wang JC, Lee SH, et al: A comparison of unilateral and bilateral laminotomies for decompression of L4-L5 spinal stenosis. Spine (Phila Pa 1976) 36: E172-178, 2011

8. Jang JW, Park JH, Hyun SJ, Rhim SC: Clinical outcomes and radiologic changes following microsurgical bilateral decompression via a unilateral approach in patients with lumbar canal stenosis and grade I degenerative spondylolisthesis with a minimum 3-year follow-up. J Spinal Disord Tech [Epub ahead of print], 2012

9. Liu X, Yuan S, Tian Y: Modified unilateral laminotomy for bilateral decompression for lumbar spinal stenosis: technical note. Spine (Phila Pa 1976) 38:E732-737, 2013

10. Mariconda M, Fava R, Gatto A, Longo C, Milano C: Unilateral laminectomy for bilateral decompression of lumbar spinal stenosis: a prospective comparative study with conservatively treated patients. J Spinal Disord Tech 15:39-46, 2002

11. Mobbs RJ, Li J, Sivabalan P, Raley D, Rao PJ: Outcomes after decompressive laminectomy for lumbar spinal stenosis: comparison between minimally invasive unilateral laminectomy for bilateral decompression and open laminectomy: clinical article. J Neurosurg Spine 21:179-186, 2014

12. Müslüman AM, Cansever T, Yılmaz A, Çavuşoğlu H, Yüce Ï, Aydın Y: Midterm outcome after a microsurgical unilateral approach for bilateral decompression of lumbar degenerative spondylolisthesis. J Neurosurg Spine 16:68-76, 2012

13. Nasca RJ: Surgical management of lumbar spinal stenosis. Spine (Phila Pa 1976) 12:809-816, 1987

14. Oertel MF, Ryang YM, Korinth MC, Gilsbach JM, Rohde V: Long-term results of microsurgical treatment of lumbar spinal stenosis by unilateral laminotomy for bilateral decompression. Neurosurgery 59:1264-1269, 2006

15. Phan K, Mobbs RJ: Minimally invasive versus open laminectomy for lumbar stenosis: a systematic review and meta-analysis. Spine (Phila Pa 1976) 41:E91-E100, 2016

16. Spetzger U, Bertalanffy H, Reinges MH, Gilsbach JM: Unilateral laminotomy for bilateral decompression of lumbar spinal stenosis. Part II: Clinical experiences. Acta Neurochir (Wien) 139: 397-403, 1997

17. Thomé C, Zevgaridis D, Leheta O, Bäzner H, Pöckler-Schöniger C, Wöhrle J, et al: Outcome after less-invasive decompression of lumbar spinal stenosis: a randomized comparison of unilateral laminotomy, bilateral laminotomy, and laminectomy. J Neurosurg Spine 3:129-141, 2005

18. Usman M, Ali M, Khanzada K, Ishaq M, Naeem-ul-Haq, Aman $\mathrm{R}$, et al: Unilateral approach for bilateral decompression of lumbar spinal stenosis: a minimal invasive surgery. J Coll Physicians Surg Pak 23:852-856, 2013

19. Yaman O, Ozdemir N, Dagli AT, Acar E, Dalbayrak S, Temiz C: A comparison of bilateral decompression via unilateral approach and classic laminectomy in patients with lumbar spinal stenosis: a retrospective clinical study. Turk Neurosurg 25:239-245, 2015

20. Zhang L, Miao HX, Wang Y, Chen AF, Zhang T, Liu XG: Limited unilateral decompression and pedicle screw fixation with fusion for lumbar spinal stenosis with unilateral radiculopathy: a retrospective analysis of 25 cases. J Korean Neurosurg Soc 58:65-71, 2015 